University of Nebraska - Lincoln

DigitalCommons@University of Nebraska - Lincoln

$9-22-2000$

\title{
Ice nanotube: What does the unit cell look like?
}

\author{
Kenichiro Koga \\ Fukuoka University of Education, koga@cc.okayama-u.ac.jp \\ Ruben D. Parra \\ University of Nebraska-Lincoln, rparra1@depaul.edu \\ Hideki Tanaka \\ Okayama University, htanakaa@cc.okayama-u.ac.jp \\ Xiao Cheng Zeng \\ University of Nebraska-Lincoln, xzeng1@unl.edu
}

Follow this and additional works at: https://digitalcommons.unl.edu/chemzeng

Part of the Chemistry Commons

Koga, Kenichiro; Parra, Ruben D.; Tanaka, Hideki; and Zeng, Xiao Cheng, "Ice nanotube: What does the unit cell look like?" (2000). Xiao Cheng Zeng Publications. 50.

https://digitalcommons.unl.edu/chemzeng/50

This Article is brought to you for free and open access by the Published Research - Department of Chemistry at DigitalCommons@University of Nebraska - Lincoln. It has been accepted for inclusion in Xiao Cheng Zeng Publications by an authorized administrator of DigitalCommons@University of Nebraska - Lincoln. 


\title{
Ice nanotube: What does the unit cell look like?
}

\author{
Kenichiro Koga \\ Department of Chemistry, Fukuoka University of Education, Fukuoka 811-4192, Japan \\ Ruben D. Parra \\ Department of Chemistry and Center for Materials \& Analysis, University of Nebraska, \\ Lincoln, Nebraska 68588 \\ Hideki Tanaka \\ Department of Chemistry, Okayama University, Okayama 700-8530, Japan \\ X. C. Zenga) \\ Department of Chemistry and Center for Materials \& Analysis, University of Nebraska, \\ Lincoln, Nebraska 68588
}

(Received 1 June 2000; accepted 28 June 2000)

\begin{abstract}
It is discovered that for an $n$-gonal ice nanotube built from stacking a single type of $n$-gonal rings of water, the unit cell consists of two stacked $n$-gonal rings. In one ring the $\mathrm{O}-\mathrm{H}$ bonds of water molecules line up clockwise whereas in the other ring the $\mathrm{O}-\mathrm{H}$ bonds line up counterclockwise. Among the $n$-gonal ice nanotubes examined, the pentagonal or hexagonal ice nanotube appears to be the most stable. (C) 2000 American Institute of Physics. [S0021-9606(00)51036-8]
\end{abstract}

A quasi-one dimensional (Q1D) crystal can be built by stacking periodically a "structure motif," i.e., the unit cell, along one direction. Such a Q1D crystal can be formed in a nanopore, e.g., a zeolite ${ }^{1,2}$ or carbon nanotube. ${ }^{3,4}$ An important class of Q1D nanocrystals is an infinitely long Q1D superlattice, or a nanotube, produced by periodically stacking a single $n$-gonal ring of molecules, e.g., a square ( $n$ $=4)$, a pentagonal $(n=5)$, or a hexagonal $(n=6)$ ring along the axial direction. In this paper, we report results of a computational study of the unit cell structure and relative stability of ice nanotubes built from $n$-gonal rings of water.

To seek the unit cell structure of the ice nanotubes, we took advantage of two new "ice rules," which are naturally derived from the original ice rules. ${ }^{5}$ Rule I: Those $\mathrm{O}-\mathrm{H}$ bonds oriented along the axis of the nanotube (defined as the $z$ axis) and located at the same vertex of every $n$-gonal ring must point to the same direction, either parallel (defined as $z^{\uparrow}$ alignment) or antiparallel (defined as $z^{\downarrow}$ alignment) with the $z$ axis [see Fig. 1(A)]. Rule II: Those $n \mathrm{O}-\mathrm{H}$ bonds in the plane of each $n$-gonal ring line up either clockwise (line-up A) or counterclockwise (line-up B), as shown in Fig. 1(B). For the above-described ice nanotube, these two rules are compulsory when every water molecule is required to be a double donor and a double acceptor of hydrogen bonds. As a result, the infinitely long hydrogen-bonded nanotube contains no defect.

The enforcement of the two new ice rules greatly reduces the number of possible structures for a finite-size $n$-gonal ice tubule. For a tubule consisting of $m$ number of stacked $n$-gonal rings (having $m \times n \mathrm{H}_{2} \mathrm{O}$ molecules), the upper limit of possible structures, $N_{m n}$, can be determined via

$$
N_{m n}=2^{m-1} 2^{n-1},
$$

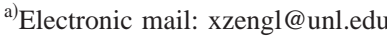

where $2^{m-1}$ and $2^{n-1}$ are the number of ways to assign A or B line-up to the $m n$-gonal rings and $z^{\uparrow}$ or $z^{\downarrow}$ alignment at the $n$ vertices of each ring. Additionally, it turns out from potential-energy calculations that a $z^{\uparrow}$ alignment of $\mathrm{O}-\mathrm{H}$ bonds favors a $z$ alignment as its nearest neighbor. Thus $2^{n-1}$ ways to set up $z$ alignments are not equally likely but only one of them is most favored, which has a minimal number of $z^{\uparrow}-z^{\uparrow}$ or $z^{\downarrow}-z^{\downarrow}$ nearest pairs. This may be termed an elective ice rule. The elective rule is best satisfied for any stacked $n$-gons with $n$ even, whereby no $z^{\uparrow}-z^{\uparrow}$ or $z^{\downarrow}-z^{\downarrow}$ nearest pair exists and the net dipole moment of the ice tubule or Q1D ice crystal is zero. When $n$ is odd (e.g., stacked pentagons), on the other hand, one $z^{\uparrow}-z^{\uparrow}$ (or $z^{\downarrow}-z^{\downarrow}$ ) nearest pair is unavoidable. But in any case, the implementation of the elective rule further reduces the number of structures, $N_{m n}$, to $N_{m}=2^{m-1}$. In Table I, we list those finite-size $n$-gonal ice tubules examined in this study. Because of the symmetry, the actual number of independent structures is generally less than $N_{m}$. For example, a square tubule built from four $(m=4)$ stacked squares has $8\left(=N_{m}\right)$ possible structures under the constraint of two compulsory and one elective rules. However, the actual number of independent structures is only 6, as shown in Fig. 1(C).

For each class ( $m$ stacked rings) of the finite-size $n$-gonal ice tubule listed in Table I, we explored the lowest-energy structure among the $N_{m}$ ones by minimizing the potential energy of each structure. ${ }^{6-8}$ We adopted two potential models of water, the Carravetta-Clementi (CC) potential ${ }^{9}$ and the TIP4P potential. ${ }^{10}$ The former is fitted to the two-body interaction potential energies calculated by ab initio molecular orbital methods; the latter is parametrized to reproduce the measured properties of bulk water at ambient temperature and pressure, thus effectively including certain many-body interactions. The zero-point vibration energy $E_{\mathrm{zpv}}$ was also evaluated by means of the normal mode analysis. ${ }^{11,12} E_{\text {zpv }}$ 
(A)

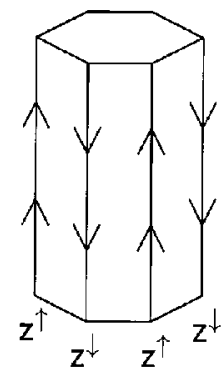

(B)
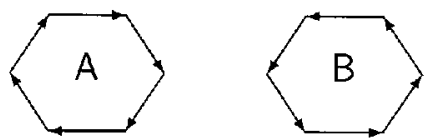

(C)

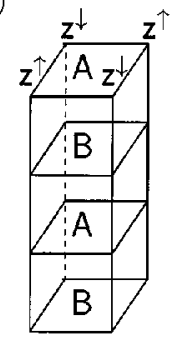

S1

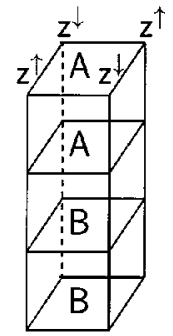

S4

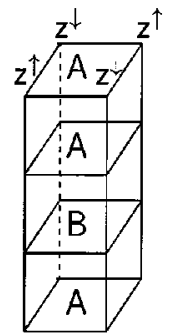

$\mathrm{S} 2$

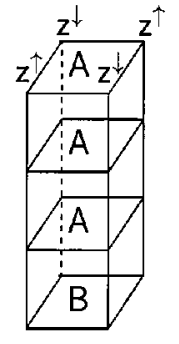

S5

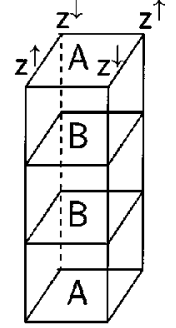

S3

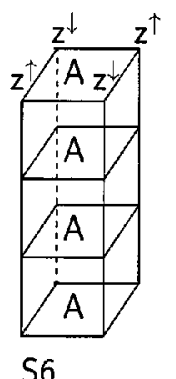

S6
FIG. 1. (A) A schematic illustration of the $z^{\uparrow}$ alignment and $z^{\downarrow}$ alignment for those $\mathrm{O}-\mathrm{H}$ bonds of water oriented along the axis of the ice nanotube. (B) A schematic illustration of A and B line-up for those $\mathrm{O}-\mathrm{H}$ bonds of water in the plane of a hexagonal ring. Line-up A refers to a clockwise orientation whereas line-up B refers to a counterclockwise orientation. (C) Schematic plots of the six independent structures for an ice tubule consisting of four stacked square rings with various A and B line-ups (see Table II also). The two compulsory and one elective ice rules are enforced.

was then added to the minimized potential energy $E_{\text {pot }}$ to yield the total energy per molecule $E_{\text {total }}$ for every structure in the specified class of ice tubule. For example, we present in Table II the result of energy-minimization calculations for the six structures shown in Fig. 1(C). Despite of drastic dif-

TABLE I. The finite-size $n$-gonal ice tubule examined.

\begin{tabular}{lcccc}
\hline \hline \multicolumn{1}{c}{ Stacked $n$-gon } & Square & Pentagon & Hexagon & Octagon \\
\hline Number of $n$-gons, $m$ & $4-12$ & $3-10$ & $3-8$ & $2-9$ \\
Number of $\mathrm{H}_{2} \mathrm{O}, m \times n$ & $16-48$ & $15-50$ & $18-48$ & $16-72$ \\
Number of structures, $N_{m}$ & $8-2048$ & $4-512$ & $4-128$ & $2-256$ \\
\hline \hline
\end{tabular}

TABLE II. The minimized energy in ascending order for the six structures (S1-S6) of four stacked square rings of water shown in Fig. 1C. Both high-level $a b$ initio and empirical potential calculations give the same lowest-energy structure, S1, under the constraint of two compulsory and one elective ice rules.

\begin{tabular}{ccccc}
\hline \hline Ab initio & Stacking type & Point group & CC & TIP4P \\
\hline$E_{\mathrm{S} 1}$ & $\mathrm{ABAB}$ & $D_{2}$ & $E_{\mathrm{S} 1}$ & $E_{\mathrm{S} 1}$ \\
$E_{\mathrm{S} 2}$ & $\mathrm{AABA}$ & $C_{2}$ & $E_{\mathrm{S} 2}$ & $E_{\mathrm{S} 2}$ \\
$E_{\mathrm{S} 3}$ & $\mathrm{ABBA}$ & $S_{4}$ & $E_{\mathrm{S} 3}$ & $E_{\mathrm{S} 4}$ \\
$E_{\mathrm{S} 4}$ & $\mathrm{AABB}$ & $D_{2}$ & $E_{\mathrm{S} 4}$ & $E_{\mathrm{S} 3}$ \\
$E_{\mathrm{S} 5}$ & $\mathrm{AAAB}$ & $C_{2}$ & $E_{\mathrm{S} 5}$ & $E_{\mathrm{S} 5}$ \\
$E_{\mathrm{S} 6}$ & $\mathrm{AAAA}$ & $S_{4}$ & $E_{\mathrm{S} 6}$ & $E_{\mathrm{S} 6}$ \\
\hline \hline
\end{tabular}

ferences in functional form between the CC and TIP4P potential models of water, we find, interestingly, that the energy minimization using both potential models yields the same lowest minimum-energy structure-the S1 structure in which the four stacked squares have $A B A B$ in-plane $\mathrm{O}-\mathrm{H}$ bond line-ups.

We also carried out energy minimization and zero-point energy calculation using high-level ab initio molecular orbital methods ${ }^{13}$ to confirm the result from potential-energy calculations. The electronic energy of every structure was calculated at the MP2/6-31+G $(d)$ level whereas the zeropoint energy was calculated at B3LYP/6-31+G(d) level. Both calculations were based on the structure optimized at the B3LYP/6-31+ $\mathrm{G}(d)$ level. As shown in Table II, the $a b$ initio calculation indeed yields the same lowest-energy structure as that from both TIP4P and CC potentials, that is, the $\mathrm{S} 1$ structure with ABAB line-ups. Moreover, the CC potential yields exactly the same ordering of six minimum-energy structures compared with the ab initio calculation. This is somewhat surprising because the CC potential is purely a two-body potential fitted to ab initio calculation. It would be interesting to examine another water potential, the $\mathrm{NCC}^{14}$ potential, which is also fitted to $a b$ initio calculation but includes explicitly the many-body interactions. Perhaps the most surprising result is that the energy-minimization calculations for all (about 6800) structures listed in Table I give rise to the same type of lowest-energy structure-the S1-like structure with $\mathrm{ABAB}$-type of ring stacking, irrespective to the number of rings, $m$, and the potential models of water (CC and TIP4P). In Figs. 2(A)-2(D), we show typical lowest-energy structures for stacked square, pentagonal, hexagonal and octagonal rings (composed of $48-50 \mathrm{H}_{2} \mathrm{O}$ molecules), respectively.

This remarkable uniformity of the way of ring stacking in all the lowest-energy structures suggests that the selection of $\mathrm{ABAB}$ stacking is perhaps a generic feature for any $m$ stacked $n$-gonal rings of water. This may also imply that in the finite-size tubule to Q1D crystal limit (i.e., $m \rightarrow \infty$ ) this ABAB-type of ring stacking may still be preserved. To examine this possibility, we carried out further energyminimization and zero-point energy calculation for a number of periodic superlattice of ice tubules (i.e., ice nanotubes). The unit cell of these nanotubes consists of three to eight stacked $n$-gonal rings of water with all the possible ways of 

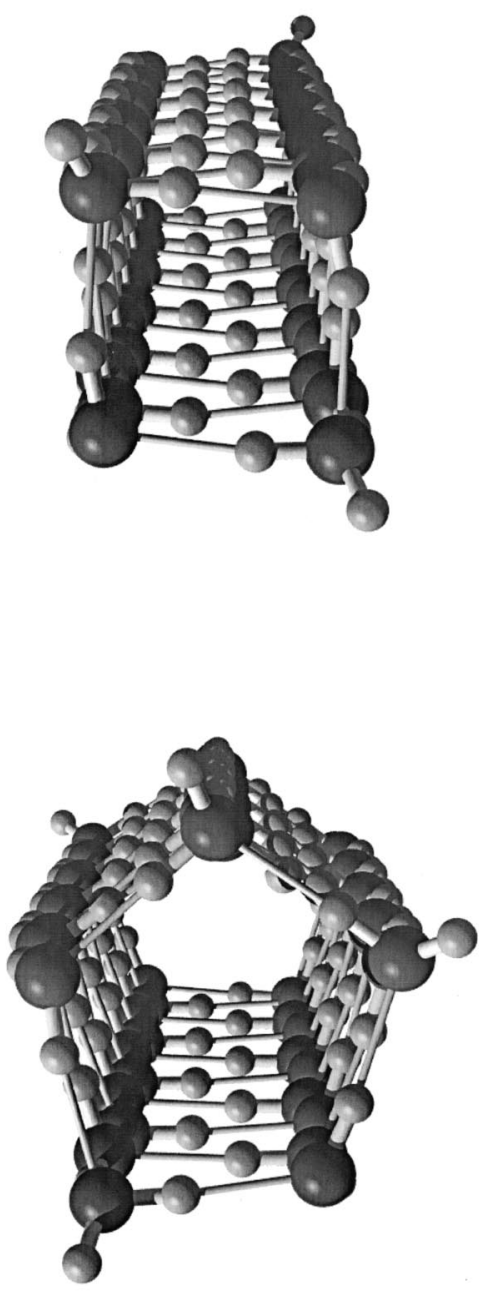

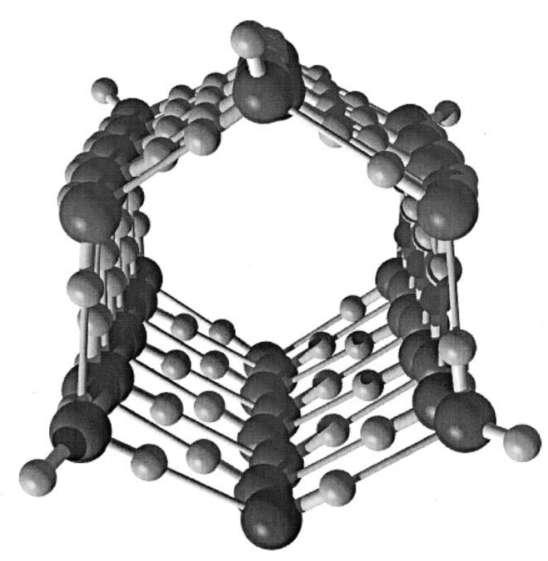

FIG. 2. Typical lowest-energy structures for stacked (A) square, (B) pentagonal, (C) hexagonal, and (D) octagonal rings, composed of 48-50 $\mathrm{H}_{2} \mathrm{O}$ molecules. The ABAB stacking is shown in all structures. arranging A and B line-ups. Among all the possible arrangements examined, indeed, we find that the ice nanotube built from the unit cell with the ABAB stacking always has the lowest energy per molecule. One may question why the ABAB unit cell always leads to the lowest-energy ice nanotube. To obtain a deeper insight on the molecular level, we show in Table III a comparison of various contributions to the total energy per molecule for the two TIP4P ice nanotubes built from $\mathrm{ABAB}$ unit cell and $\mathrm{AABB}$ unit cell, respectively. We note that the ice nanotube built from the ABAB unit cell always has the lowest hydrogen-bonding interaction energy. In other words, $\mathrm{ABAB}$ stacking is energetically the most favorable in the formation of the hydrogen-bond network of ice nanotube, regardless of the type of $n$-gonal ring of water and the potential model of water. Other types of stacking such as AABB are less favored in the formation of hydrogen-bond network but can be more favored by the long-ranged electrostatic interaction. However, the latter contribution plays a lesser role than that from the shortranged hydrogen-bonding interaction.

We also investigated the relative stability of square, pentagonal, hexagonal, and octagonal ice nanotubes. In Fig. 3, we plot total energy per molecule, $E_{\text {total }}$, as a function of the inverse of total number of molecules, $1 / N$, where $N$ ranges from 15 (finit-size superlattice) to $\infty$ (infinitely long Q1D crystal). All the finite-size superlattices and Q1D crystals are built from the ABAB unit cell. Both Figs. 3(A) (based on the CC potential) and 3(B) (based on the TIP4P potential) show that the octagonal superlattices have a much higher energy

TABLE III. Comparison of various contributions to the total energy per molecule for the two infinitely long ice nanotubes built from the unit-cell with $\mathrm{ABAB}$ and $\mathrm{AABB}$ stacking, respectively.

\begin{tabular}{cccccr}
\hline \hline Unit cell & $E_{\text {total }}$ a & $E_{\text {zpv }}$ & $E_{\text {pot }}$ & $E_{\mathrm{HB}}$ & \multicolumn{1}{c}{$E_{\mathrm{LR}}$} \\
\hline ABAB (sq) & -32.68 & 14.39 & -47.07 & -47.34 & 0.27 \\
AABB (sq) & -32.58 & 14.44 & -47.02 & -43.45 & -3.56 \\
& & & & & \\
ABAB (pen) & -33.56 & 14.37 & -47.94 & -46.59 & -1.34 \\
AABB (pen) & -33.46 & 14.42 & -47.88 & -42.38 & -5.49 \\
ABAB (hex) & -33.61 & 14.19 & -47.80 & -48.19 & 0.40 \\
AABB (hex) & -33.49 & 14.24 & -47.73 & -43.76 & -3.97 \\
ABAB (oct) & -33.13 & 13.84 & -46.97 & -42.34 & -4.62 \\
AABB (oct) & -32.99 & 13.90 & -46.88 & -40.56 & -6.32 \\
\hline \hline
\end{tabular}

${ }^{\mathrm{a}} E_{\mathrm{total}}=E_{\mathrm{zpv}}+E_{\mathrm{pot}}$ and $E_{\mathrm{pot}}=E_{\mathrm{HB}}+E_{\mathrm{LR}} \cdot E_{\mathrm{total}}$ is the total energy, $E_{\mathrm{zpv}}$ is the zero-point vibration energy, $E_{\mathrm{pot}}$ is the minimized potential energy, $E_{\mathrm{HB}}$ is the hydrogen-bonding interaction energy, and $E_{\mathrm{LR}}$ the long-range potential energy. All energies are in units of $\mathrm{kJ} / \mathrm{mol}$. 

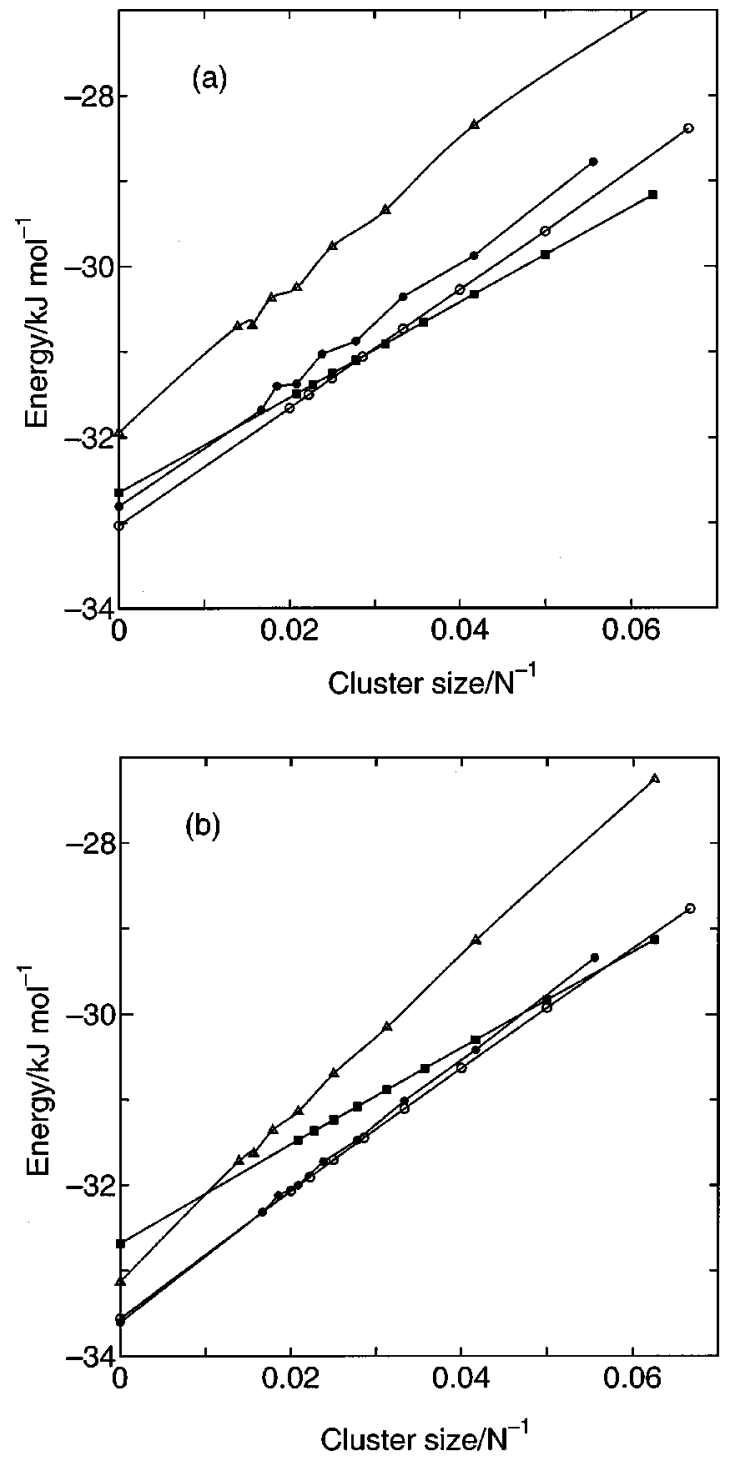

FIG. 3. Total energy per molecule vs the inverse of number of $\mathrm{H}_{2} \mathrm{O}$ molecules for stacked square (closed square), pentagonal (open circle), hexagonal (closed circle), and octagonal (triangle) rings, using (A) the CC potential model and (B) the TIP4P potential model. All finite-size ice superlattices and the infinitely long Q1D ice crystal are built from the $A B A B$ unit cell.

per molecule than the other three. A similar result would be expected for any $n$-gonal ice nanotube when $n \geqslant 7$ or $n=3$ because in those cases the internal angles of the $n$-gonal ring are either much greater or smaller than the $\mathrm{HOH}$ angle of water $\left(\sim 104^{\circ}\right)$, causing appreciable strain in the hydrogen bonds and thus a higher hydrogen-bonding energy per molecule (see the $E_{\mathrm{HB}}$ column in Table III). On the other hand, square superlattices are energetically more favorable when $N$ is small. In the $N \rightarrow \infty$ (infinitely long ice nanotube) limit, however, both pentagonal and hexagonal ice nanotubes become energetically more favorable than the square one. Interestingly, we find that with the CC model of water the pentagonal ice nanotube is more stable whereas with the TIP4P model of water the hexagonal ice nanotube is more stable. In both cases, the total energies of pentagonal and hexagonal ice nanotubes are very close to each other. Consequently, the definite determination of the relative stability between the pentagonal and hexagonal ice nanotube requires a total-energy calculation using quantum mechanical methods. ${ }^{15}$ Note in passing that in this study the temperature was not considered (zero kelvin condition). At a finite temperature, however, the energy difference between the hexagonal and pentagonal ice nanotubes may be even harder to distinguish if stacking faults are present in the ice nanotubes.

K.K. and H.T. are supported by the Japan Ministry of Education. X.C.Z. is supported by US NSF and ONR, and by a JSPS fellowship. Part of this work was completed utilizing the Research Computing Facility of the University of Nebraska-Lincoln.

${ }^{1}$ R. Radhakrishnan, K. E. Gubbins, A. Watanabe, and K. Kaneko, J. Chem. Phys. 111, 9058 (1999).

${ }^{2}$ K. Morishige and K. Kawano, J. Chem. Phys. 110, 4867 (1999).

${ }^{3}$ W. Q. Han, S. S. Fan, Q. Q. Li, and Y. D. Hu, Science 277, 1287 (1997).

${ }^{4}$ Q. Jiang, N. Aya, and F. G. Shi, Appl. Phys. A: Mater. Sci. Process. 64, 627 (1997).

${ }^{5}$ D. Eisenberg and W. Kauzmann, The Structure and Properties of Water (Oxford University Press, London, 1969).

${ }^{6}$ F. H. Stillinger and T. A. Weber, Science 225, 983 (1984).

${ }^{7}$ C. J. Tsai and K. D. Jordan, J. Phys. Chem. 97, 5208 (1993).

${ }^{8}$ D. J. Wales and I. Ohmine, J. Chem. Phys. 98, 7245 (1993).

${ }^{9}$ V. Carravetta and E. Clementi, J. Chem. Phys. 81, 2646 (1984).

${ }^{10}$ W. L. Jorgensen, J. Chandrasekhar, J. D. Madura, R. W. Impey, and M. L. Klein, J. Chem. Phys. 79, 926 (1983).

${ }^{11}$ A. Pohorille, L. R. Pratt, R. A. LaViolette, M. A. Wilson, and M. A. MacElroy, J. Chem. Phys. 87, 6070 (1987).

${ }^{12}$ I. Ohmine, H. Tanaka, and P. G. Wolynes, J. Chem. Phys. 89, 5852 (1988).

${ }^{13}$ Gaussian 98, Revision A.7, M. J. Frisch, G. W. Trucks, H. B. Schlegel et al., Gaussian, Inc. Pittsburgh, PA (1998).

${ }^{14}$ U. Niesar, G. Corongiu, E. Clementi, G. R. Kneller, and D. K. Bhattacharya, J. Phys. Chem. 94, 7949 (1990).

${ }^{15}$ C. Cavazzoni, G. L. Chiarotti, S. Scandolo, E. Tosatti, M. Bernasconi, and M. Parrinello, Science 283, 44 (1999). 